\title{
Quasi-shuffle algebras in non-commutative stochastic calculus
}

\author{
Kurusch Ebrahimi-Fard, Frédéric Patras
}

\begin{abstract}
This chapter is divided into two parts. The first is largely expository and builds on Karandikar's axiomatisation of Itô calculus for matrix-valued semimartingales. Its aim is to unfold in detail the algebraic structures implied for iterated Itô and Stratonovich integrals. These constructions generalise the classical rules of Chen calculus for deterministic scalar-valued iterated integrals. The second part develops the stochastic analog of what is commonly called chronological calculus in control theory. We obtain in particular a pre-Lie Magnus formula for the logarithm of the Itô stochastic exponential of matrix-valued semimartingales.
\end{abstract}

\section{Introduction}

Algebra, renormalisation theory as well as numerical analysis are among a range of disparate fields that have seen a surge in interest for the study of various algebraic and combinatorial structures originating from the integration by parts formula, foundational to integral calculus. In particular, the theories of Rota-Baxter algebras, shuffle and quasi-shuffle products, pre- and post-Lie algebras as well as combinatorial bialgebras on rooted trees and words have undergone expansive phases in the last two decades. The following rather incomplete list of references provides some examples of these developments $[11,17,15,20,37,35,56,62,63,65,66,69]$. In the particular context of stochastic integration, interest in these structures concentrated largely in Lyon's seminal theory of rough paths [57, 58], which is based on Chen's iterated path integrals and shuffle algebra on words [21, 22, 71]. Gubinelli expanded

Kurusch Ebrahimi-Fard

Department of Mathematical Sciences, Norwegian University of Science and Technology - NTNU, 7491 Trondheim, Norway, e-mail: kurusch.ebrahimi-fard@ntnu.no, https://folk.ntnu.no/kurusche/

Frédéric Patras

Université Côte d'Azur, Labo. J.-A. Dieudonné, UMR 7351, CNRS, Parc Valrose, 06108 Nice

Cedex 02, France, e-mail: patras@unice.fr,www-math.unice.fr/ patras 
Lyon's theory by generalising it to a certain combinatorial Hopf algebra of rooted trees. The resulting notion of branched rough paths [40, 41, 45] draws inspiration from Butcher's theory of B-series in numerical integration of differential equations $[42,59]$ as well as Connes and Kreimer's Hopf algebraic approach to renormalisation in perturbative quantum field theory [23]. The latter, moreover emphasised the pre-Lie algebraic perspective on rooted trees [19, 61]. Ideas from rough paths gave rise to various new developments, culminating in Hairer's celebrated theory of regularity structures $[43,44]$ and its algebraic renormalisation theory [12] used in the construction of solutions of very irregular S(P)DEs.

The relevance of such algebraic structures for classical - non-commutative stochastic integration (in the sense of Itô-Stratonovich) [6, 70] has attracted less attention. Foundational papers in this field are Gaines's 1994 work on the algebra of iterated stochastic integrals [39], introducing what is now called quasi-shuffle product, as well as the equivalent sticky shuffle product formula for iterated quantum Itô integrals introduced in 1995 in the context of quantum stochastic calculus [8]. We refer to Hudson's review papers on Hopf-algebraic aspects of iterated stochastic integrals $[48,49]$. The authors together with Charles Curry, Alexander Lundervold, Simon Malham, Hans Munthe-Kaas and Anke Wiese further developed the use of (quasi-)shuffle algebra in stochastic integration theory and numerical methods for SDEs in several joint works [24, 25, 26, 29, 32, 33].

The present article is divided into two main parts. The first part starts by recalling Karandikar's axiomatisation of Itô calculus for matrix-valued continuous semimartingales [52]. We discuss in detail the algebraic structures implied for iterated Itô and Stratonovich integrals. To the best of our knowledge, such an account does not seem to exist in the literature. In fact, Karandikar's ideas do not seem to be widely known, as the algebraic notions and techniques involved are not of common use in non-commutative stochastic calculus. Our presentation is written with a view toward operationality and therefore, as far as possible, in the language of theoretical probability theory. In modern algebraic terminology, Karandikar's axioms define the notion of non-commutative quasi-shuffle algebra. We proceed by building consistently on this structure aiming at unraveling properties of integration techniques, ranging from general matrix-valued semimartingales to more specific situations (continuous paths). We remark that introducing the Stratonovich integral in full generality requires extra axioms corresponding to the splitting of the quadratic covariation bracket into a continuous and a jump part. We hope that these ideas might be useful also in other settings.

The second part develops the stochastic analog of what is commonly called chronological calculus in control theory $[1,4,5]$. We feature in particular Agrachev and Gamkrelidze's [2,3] study and systematical use of chronological algebra in control problems. In mathematics, chronological algebras are known as pre-Lie or Vinberg algebras $[13,17]$. This part of our work is a continuation of joint work with Charles Curry [25], where a pre-Lie Magnus formula for the logarithm of the Stratonovich stochastic exponential for continuous matrix-valued semimartingales was introduced. 
A more detailed outline of the paper follows. The first section is divided into three subsections. We begin by focusing on general Itô calculus for matrix-valued semimartingales, introduce Karandikar's axioms, and point out connections with other theories, especially Rota-Baxter algebras. The second subsection introduces formally the splitting of the covariation bracket, leading to a tentative axiomatisation of Stratonovich calculus. The last subsection studies stochastic calculus for continuous semimartingales, a situation where the axioms simplify dramatically, allowing to replace quasi-shuffles by shuffles, that is, permitting the use the standard rules of calculus.

In the second section we study pre-Lie algebraic aspects in stochastic integral calculus. We consider the pre-Lie Magnus formula in the general context of enveloping algebras of pre-Lie algebras - the corresponding section can be understood also as an introduction to pre-Lie structures since we survey some of their most relevant properties for general integral calculus, following the chapter [34] written in the context of classical integration. The second and last subsection of this section changes focus by developing instead a pre-Lie point of view on Itô integral calculus, without restriction to continuous matrix-valued semimartingales. We obtain in particular a pre-Lie Magnus formula for the logarithm of the Itô stochastic exponential of matrix-valued semimartingales.

Conventions i) With the aim of simplifying the presentation we shall always assume that the value of semimartingales is zero at $t=0$. ii) All structures are defined over a ground field $k$ of characteristic zero.

Acknowledgements The second author would like to express his gratitude for the warm hospitality he experienced during the 2019 Verona meeting, Random transformations and invariance in stochastic dynamics, with special thoughts for S. Albeverio and S. Ugolini. This work was supported by the French government, managed by the ANR under the UCA JEDI Investments for the Future project, reference number ANR-15-IDEX-01.

\section{Karandikar's axioms and quasi-shuffle algebras}

\subsection{Itô calculus for semimartingales}

This section discusses the formal properties of the integral calculus for semimartingales. Protter's textbook [70] will serve as the standard reference on stochastic integration. The central aim is to feature Karandikar's axioms for matrix-valued Itô integrals and the corresponding notion of non-commutative quasi-shuffle algebra ${ }^{1}$.

Recall first Itô's integration by parts formula for scalar semimartingales $X, Y[70$, chapter II.6]

\footnotetext{
${ }^{1}$ Karandikar's axioms appeared in a 1982 paper [52]. They were (re-)discovered almost two decades later in a completely different context -the one of Stasheff polytopes- as axioms for dendriform trialgebras [55].
} 


$$
X_{t} Y_{t}=\int_{0}^{t} X_{s^{-}} d Y_{s}+\int_{0}^{t} Y_{s^{-}} d X_{s}+[X, Y]_{t} .
$$

Here as well as in the rest of the paper, our conventions are in place, that is, we assume that $X_{0}=Y_{0}=0$. Equation (1) defines the so-called quadratic covariation bracket, $[X, Y]_{t}$, the extra term that distinguishes Itô's integration by parts formula from the classical one.

To deal with matrix-valued semimartingales, one has to take into account the non-commutativity of matrix multiplication. The product in the second term on the righthand side of (1) is then in the wrong order. Following Protter (and Karandikar), we introduce left and right stochastic integrals

$$
(X>Y)_{t}:=\int_{0}^{t} X_{s^{-}} d Y_{s} \quad(X \prec Y)_{t}:=\int_{0}^{t} d X_{S} Y_{s^{-}} .
$$

Itô's integration by parts formula for matrix-valued semimartingales $X, Y$ writes then [70, chapter V.8, Thm. 47]

$$
X_{t} Y_{t}=(X>Y)_{t}+(X \prec Y)_{t}+[X, Y]_{t},
$$

Notice that in the case of scalar-valued semimartingales we have that $X<Y=Y>X$ such that (3) can be changed back to (1).

Remark 1. Protter and Karandikar use a different notation: $(X>Y)_{t}=(X \cdot Y)_{t}=$ and $(X \prec Y)_{t}=(X: Y)_{t}$. Our notation is in line with the one used in algebra. It is also convenient to identify the time-ordering of operations, see [34].

Hereafter, we account for various properties of (left and right) stochastic integrals of scalar-valued semimartingales. Even though some of the ternary formulas considered may seem redundant in the scalar case, we emphasise that they do not involve permutations of the variables. They thus hold immediately for $(n \times n)$ matrix-valued semimartingales.

Recall first [70, Chapter II.6, Thm. 29] that for $H, K$ adapted processes with caglad (left continuous with right limits) paths and $X, Y$ two semimartingales we have $\left[\int_{0}^{t} H_{s} d X_{s}, \int_{0}^{t} K_{s} d Y_{s}\right]_{t}=\int_{0}^{t} H_{S} K_{s} d[X, Y]_{s}$.

Assuming now that $L$ and $M$ are semimartingales, we get $[L>X, M>Y]=$ $(L M)>[X, Y]$ which simplifies to the ternary relation

$$
[L>X, Y]=L>[X, Y] .
$$

Similarly, $[X \prec Y, Z]=[X, Y>Z]$ and $[X, Y] \prec Z=[X, Y<Z]$.

Other ternary relations satisfied by semimartingales follow directly from standard properties and from the definitions:

$$
(X>(Y>Z))_{t}=\int_{0}^{t} X_{s^{-}} d\left(\int_{0}^{t} Y_{s^{-}} d Z_{s}\right)=\int_{0}^{t} X_{s^{-}} Y_{s^{-}} d Z_{s}=((X Y)>Z)_{t} .
$$


Similarly, for $G, H$ caglad and $Y$ a semimartingale, $\int_{0}^{t} G_{s} d\left(\int_{0}^{t} H_{s} d Y_{s}\right)=\int_{0}^{t} G_{s} H_{s} d Y_{s}$ [70, Thm. II.19]. Dually, $(X<Y)<Z=X<(Y Z)$. Finally,

$$
((X>Y)<Z)_{t}=\int_{0}^{t}\left(d \int_{0}^{s} X_{u^{-}} d Y_{u}\right) Z_{s^{-}}=\int_{0}^{t} X_{s^{-}} d Y_{s} Z_{s^{-}}=(X>(Y<Z))_{t} .
$$

Notice that the associativity of the quadratic covariation bracket, $[X,[Y, Z]]=$ $[[X, Y], Z]$, can be deduced from the associativity of the usual product of semimartingales together with the previous identities:

$$
\begin{aligned}
& {[[X, Y], Z]=[X Y-X<Y-X>Y, Z]} \\
& =(X Y) Z-(X Y)<Z-(X Y)>Z-[X<Y+X>Y, Z] \\
& =X(Y Z)-X<(Y Z)-X>(Y<Z)-X>(Y>Z) \\
& \quad-X>[Y, Z]-[X, Y<Z]-[X, Y>Z] \\
& =[X, Y Z]-[X, Y<Z]-[X, Y>Z]=[X,[Y, Z]] .
\end{aligned}
$$

Note that, as mentioned before, $X, Y$ and $Z$ appear always in the same order in the above formulas. This implies that they hold in the matrix-valued case. In that case, $[X, Y]$ is defined in terms of the component-wise quadratic covariation brackets, $[X, Y]_{i k}=\sum_{j=1}^{n}\left[X_{i j}, Y_{j k}\right]$, and similarly for the other products. Putting this together, yields the axiomatisation of Itô calculus for semimartingales, due to Karandikar.

Theorem 1 (Karandikar, [52]). The left- and right stochastic Itô integrals satisfy Karandikar's identities for matrix-valued semimartingales $X, Y, Z$

$$
\begin{aligned}
(X<Y)<Z & =X<(Y Z) \\
X>(Y>Z) & =(X Y)>Z \\
(X>Y)<Z & =X>(Y<Z) \\
(X Y) Z & =X(Y Z) \\
{[X>Y, Z] } & =X>[Y, Z] \\
{[X<Y, Z] } & =[X, Y>Z] \\
{[X, Y]<Z } & =[X, Y<Z] .
\end{aligned}
$$

Remark 2. Karandikar considered in [52] the continuous case. Axiom 10 is stated in a slightly different way - namely in the case where $Y$ is non-singular [52, Eq. (9) p. 1089]. However, these restrictions (to the continuous case and to non-singular $Y$ ) are not necessary, see Karandikar [54].

Definition 1. An associative space $A$ equipped with three products $<,>$ and $[$,$] ,$ called respectively the left half-shuffle, the right half-shuffle and the bracket, such that $X Y=X<Y+X>Y+[X, Y]$ and satisfying Karandikar's identities in Theorem 1 is called a quasi-shuffle algebra. 
See Remark 4 below for an explanation of the terminology. Karandikar's axiomatisation of Itô calculus therefore says that the algebra of semimartingales is a non-commutative quasi-shuffle algebra. There are many examples of quasi-shuffle algebras besides the one coming from stochastic calculus and Karandikar's results apply immediately to them. Conversely, general results from abstract quasi-shuffle algebra apply to stochastic calculus. Examples of application of this strategy can be found in our joint works with Simon Malham and Anke Wiese [32, 33].

Remark 3. We have seen that the associativity of the bracket operation $[$,

$$
[X,[Y, Z]]=[[X, Y], Z]
$$

follows from the associativity of product in the algebra $A$. One can define equivalently a quasi-shuffle algebra to be a vector space $A$ equipped with three products $<,>$ and $[$,$] satisfying Equations (5-11) and (8) replaced by (12), where one sets$ $X Y=X\langle Y+X>Y+[X, Y]$. The associativity of the product $X Y$ results then automatically from these axioms (the proof parallels the proof showing that the quadratic covariation bracket is associative for semimartingales):

$$
\begin{aligned}
& (X Y) Z=(X Y)<Z+(X Y)>Z+[X<Y+X>Y+[X, Y], Z] \\
& =X \prec(Y Z)+X>(Y<Z)+X>(Y>Z) \\
& \quad+[X, Y<Z]+[X, Y>Z]+X>[Y, Z]+[X,[Y, Z]] \\
& =X \prec(Y Z)+X>(Y<Z)+X>(Y>Z)+X>[Y, Z]+[X, Y Z]=X(Y Z) .
\end{aligned}
$$

Remark 4. The terminology "quasi-shuffle" algebra is used in algebra and combinatorics. It reflects the close similarity with the classical shuffle algebra [72]. The work [37] explores the relation between the two families of algebras from a deformation theoretical viewpoint. Hoffman [46], independently of Karandikar's seminal work, largely initiated the development of the Hopf algebraic theory of commutative quasi-shuffle algebra. However, as we mentioned in the introduction, Gaines [39] as well as Hudson et al. [8, 48, 49] introduced quasi-shuffle products to study properties of products of iterated Itô integrals. We note that Cartier, back in 1972 [17], used a quasi-shuffle product in the construction of free (Rota-)Baxter algebra.

When dealing with scalar-valued semimartingales, we already noticed that $X<$ $Y=Y>X$. Correspondingly, Karandikar's axioms simplify, leading to the notion of commutative quasi-shuffle algebra studied in detail by Hoffman, see [36] for a recent account. Some extra properties are then available such as Hoffman's isomorphism linking shuffle and quasi-shuffle products. Features of the commutative theory have been exploited recently in stochastic calculus, for example in [24, 33, 38].

From a purely algebraic viewpoint, the fundamental example of a quasi-shuffle algebra is the linear span of words $X=x_{1} \cdots x_{n}$ where the $x_{i}$ belong to a monoid $M$ with (not necessarily commutative) product denoted $\times$. The axioms for the products, $<,>$, and $[$,$] , used to define inductively the associative product X * Y:=X<$ $Y+X>Y+[X, Y]$ of words $X, Y$, are given by:

$$
x_{1} \cdots x_{n}<y_{1} \cdots y_{m}:=x_{1}\left(x_{2} \cdots x_{n} * y_{1} \cdots y_{m}\right)
$$




$$
\begin{aligned}
x_{1} \cdots x_{n}>y_{1} \cdots y_{m} & :=y_{1}\left(x_{1} \cdots x_{n} * y_{2} \cdots y_{m}\right) \\
{\left[x_{1} \cdots x_{n}, y_{1} \cdots y_{m}\right] } & :=\left(x_{1} \times y_{1}\right)\left(x_{2} \cdots x_{n} * y_{2} \cdots y_{m}\right) .
\end{aligned}
$$

For example, taking $M$ to be the monoid of the integers, we have the quasi-shuffle product

$$
23 \prec 1=2(3 * 1)=2(3 \prec 1+3>1+[3,1])=231+213+24 \text {. }
$$

Remark 5. In the following, quasi-shuffle algebra shall mean non-commutative quasishuffle algebra. The latter are also called tridendriform algebras in the literature. However, the quasi-shuffle terminology, besides being close to other ones that have been used in stochastics (modified shuffle product, sticky shuffle product, ...) has the advantage of underlining the connection to the familiar shuffle calculus for Chen's iterated integrals and the related product of simplices, as well as many more topics (such as quasi-symmetric functions, multizeta values, etc.). We refer to $[30,36,67,68]$ for accounts on the combinatorial theory of quasi-shuffle algebras as well as further bibliographical references and various examples. See $[24,25,32,33,39,48,49]$ and references therein for more details and references on quasi-shuffle calculus in probability.

We introduce now Rota-Baxter algebras, which provide a more general approach to the algebraic axiomatisation of integral calculus [76] and therefore an important class of examples for quasi-shuffle algebras. Indeed, Theorem 2 below shows that any Rota-Baxter algebra is a quasi-shuffle algebra [27]. We refer to the survey [34] for further details and references about Rota-Baxter algebras and their use in integral calculus, probability theory, renormalisation in perturbative quantum field theory and classical integrable systems.

Definition 2. A Rota-Baxter algebra of weight $\theta \in k$ consists of an associative $k$ algebra $A$ equipped with a linear operator $R: A \rightarrow A$ satisfying the Rota-Baxter relation of weight $\theta$ :

$$
R(x) R(y)=R(R(x) y+x R(y)+\theta x y) \quad \forall x, y \in A .
$$

Note that if $R$ is a Rota-Baxter map of weight $\theta$, then the map $R^{\prime}:=\beta R$ for $\beta \in k$ different from zero is of weight $\beta \theta$. This permits to rescale the original weight $\theta \neq 0$ to the standard weight $\theta^{\prime}=+1$ (or $\theta^{\prime}=-1$ ). The argument of the map $R$ on the righthand side of (13) consists of a sum of three terms; one can show that it defines a new associative product on $A$.

Definition 3 (Rota-Baxter product). The Rota-Baxter associative product is defined by

$$
x *_{\theta} y:=R(x) y+x R(y)+\theta x y .
$$

The Rota-Baxter relation originated in the work of the mathematician Glen Baxter [7]. Rota [73, 75] followed by Cartier [16] made important contributions to the 
algebraic foundations of Baxter's work, among others, by providing different constructions of free commutative objects. The idea of quasi-shuffle product is actually often traced back to Cartier's 1972 article.

Theorem 2 ([27]). Assume now that $\theta=1$. Writing $a \cdot b:=a b$ for the usual associative product on $A$ and setting $a<b:=a R(b)$ and $a>b:=R(a) b$, so that $*:=<+>+\cdot$, then the quasi-shuffle algebra identities hold:

$$
\begin{array}{lll}
(a<b)<c=a<(b * c), & & (a>b) \cdot c=a>(b \cdot c) \\
a>(b>c)=(a * b)>c, & & (a<b) \cdot c=a \cdot(b>c) \\
(a>b)<c=a>(b<c), & & (a \cdot b)<c=a \cdot(b<c) .
\end{array}
$$

Remark 6. Without the normalisation to the standard weight, one obtains an example of a $\theta$-quasi-shuffle algebra, studied in greater detail in [14]. See also [47].

Example 1 (Fluctuation theory). Baxter's work was motivated by problems in the theory of fluctuations [74]. The latter deals with extrema of sequences of real valued random variables. Their distribution can be studied using operators on random variables such as $X \rightarrow X^{+}:=\max (0, X)$. This motivates to define the operator

$$
R(F)(t):=\mathbf{E}\left[\exp \left(i t X^{+}\right)\right]
$$

on characteristic functions $F(t):=\mathbf{E}[\exp (i t X)]$ of real valued random variables $X$, which is a Rota-Baxter map of weight $\theta=1$.

Example 2 (Finite summation operators). On functions $f$ defined on $\mathbb{N}$ and with values in an associative algebra $A$, the summation operator $R(f)(n):=\sum_{k=0}^{n-1} f(k)$ is a Rota-Baxter map of weight one. It is the right inverse of the finite difference operator $\Delta(f)(n):=f(n+1)-f(n)$.

Remark 7 (Shuffle algebras in classical calculus). Before concluding this section, we apply the previous ideas to the case of deterministic matrix-valued semimartingales. Even in that seemingly simple case the relations put forward by Karandikar prove to be interesting and useful.

We consider for example the algebra $A$ of matrices whose entries are continuous functions of finite variation. Then, since the quadratic covariation bracket vanishes, Karandikar's identities reduce to an algebra equipped with an associative product $X Y=X \prec Y+X>Y$ and

$$
\begin{aligned}
& (X<Y)<Z=X<(Y Z) \\
& X>(Y>Z)=(X Y)>Z \\
& (X>Y)<Z=X>(Y<Z)
\end{aligned}
$$

Our previous arguments show that the associativity of the product $X Y$ can be recovered formally from these identities. These relations have been used first by Eilenberg and MacLane to give an abstract proof of the associativity of the shuffle product of simplices in topology. 
Definition 4. The three identities (16) define the structure of non-commutative shuffle algebra (aka dendriform algebra).

From Theorem 2 is clear that on a Rota-Baxter algebra of weight $\theta=0$ one can define left and right half-shuffle products satisfying the three identities (16). We refer to [34] for a survey and more details and applications in classical integral calculus as well as general references on the subject. We will come back to these relations later as they encode the algebra structure underlying Stratonovich calculus for semimartingales.

\subsection{Singular quasi-shuffle algebras and Stratonovich calculus}

In this section we extend Karandikar's axiomatisation beyond the setting of continuous semimartingales. Namely, we include in the algebraic description of Itô calculus the decomposition into continuous and jump parts of the quadratic covariation bracket [70, Chapter II.6].

We write $\Delta(X)$ for the process of jumps of a semimartingale $X$, i.e., $\Delta(X)_{s}=$ $\left(X-X_{-}\right)_{s}$, and introduce the corresponding decomposition of the bracket into continuous and jump parts, $[X, Y]=[X, Y]^{c}+[X, Y]^{j}$. The definition extends from the scalar-valued to the continuous matrix-valued case components-wise. For scalarvalued processes, $[X, X]_{t}^{j}=\sum_{0 \leqslant s \leqslant t}\left(\Delta(X)_{s}\right)^{2}$, a term that appears frequently in stochastic calculus, for example, in the study of the stochastic or Doléans-Dade exponential [70, chapter II.8, Thm. 37]. A semimartingale $X$ is called quadratic pure jump if $[X, X]=[X, X]^{j}$.

Recall first a fundamental property of $\Delta$ acting on scalar-valued processes. Since the bracket of two semimartingales has paths of finite variation on compact sets [70, chapter II, Cor.1], it is a quadratic pure jump semimartingale, that is, $[[X, Y],[X, Y]]=[[X, Y],[X, Y]]^{j}$ by $[70$, chapter II.6, Thm. 26]. This implies by $[70$, chapter II.6. Thm. 28] that for arbitrary semimartingales $X, Y, Z$ we have $[[X, Y], Z]=\sum_{0 \leqslant s \leqslant t} \Delta([X, Y])_{s} \Delta(Z)_{s}$. In particular,

$$
\begin{aligned}
{\left[[X, Y]^{c}, Z\right] } & =\left[[X, Y]^{c}, Z\right]^{c}=\left[[X, Y]^{c}, Z\right]^{j}=0, \\
{[[X, Y], Z]^{c} } & =\left[[X, Y]^{j}, Z\right]^{c}=0, \\
{[[X, Y], Z] } & =\left[[X, Y]^{j}, Z\right]=\left[[X, Y]^{j}, Z\right]^{j} .
\end{aligned}
$$

As a corollary, we notice for further use that for continuous semimartingales $[[X, Y], Z]=0$. These identities hold for matrix-valued semimartingales (since the splitting of processes into a continuous and a pure jump part is linear - it commutes with taking linear combinations of brackets).

A full axiomatisation of Itô calculus taking into account such phenomena would require the introduction of the operator $\Delta$, those identities, and most likely other aspects of standard stochastic calculus. We propose a lighter version that provides an axiomatic framework allowing to relate formally Itô and Stratonovich calculi. 
Definition 5. A singular quasi-shuffle algebra is a quasi-shuffle algebra, $(A,>,<$ $,[-,-])$, such that the associative bracket splits into $[-,-]=[-,-]^{c}+[-,-]^{j}$ and furthermore the following relations hold:

$$
\begin{aligned}
& {\left[[X, Y]^{c}, Z\right]^{c}=\left[X,[Y, Z]^{c}\right]^{c}=0} \\
& {\left[[X, Y]^{c}, Z\right]^{j}=\left[X,[Y, Z]^{c}\right]^{j}=0} \\
& {\left[[X, Y]^{j}, Z\right]^{c}=\left[X,[Y, Z]^{j}\right]^{c}=0}
\end{aligned}
$$

Notice that we also have then

$$
[X<Y, Z]^{c}=[X, Y>Z]^{c},[X<Y, Z]^{j}=[X, Y>Z]^{j} .
$$

Recall that for matrix-valued semimartingales, the (left/right) Fisk-Stratonovich integrals are defined in terms of the Itô integral by

$$
\begin{aligned}
(X \geqslant Y)_{t} & :=\int_{0}^{t} X_{s} \circ d Y_{s}:=\int_{0}^{t} X_{s} d Y_{s}+\frac{1}{2}[X, Y]_{t}^{c} \\
(Y \leqslant X)_{t} & :=\int_{0}^{t} \circ d Y_{S} X_{s}:=\int_{0}^{t} d Y_{S} X_{S}+\frac{1}{2}[Y, X]_{t}^{c} .
\end{aligned}
$$

Formally, in any singular quasi-shuffle algebra one can define the two products

$$
X \geqslant Y:=X>Y+\frac{1}{2}[X, Y]^{c}, \quad X \leqslant Y:=X<Y+\frac{1}{2}[X, Y]^{c} .
$$

Then the integration by parts rule reads:

$$
X Y=X \geqslant Y+X \leqslant Y+[X, Y]^{j} .
$$

Unfortunately, it seems to be difficult to find a simpler axiomatic framework than the one of singular quasi-shuffle algebras to account for Stratonovich calculus in the presence of jumps. Indeed, it is likely that a meaningful system of ternary relations involving only $\geqslant, \leqslant$ and $[,]^{j}$ is unavailable. Fortunately, these issues simplify considerably for continuous semimartingales.

\subsection{Shuffle algebra and continuous semimartingales}

As we mentioned previously, stochastic integration simplifies dramatically when considering continuous semimartingales. The reason for this should be clear from our previous developments, that is, the jump part, $[-,-]^{j}$, of the bracket, $[-,-]$, vanishes, so that the latter reduces to its continuous part and becomes nilpotent of order 3: $[X,[Y, Z]]=[[X, Y], Z]=0$. In that situation, the axioms of Itô calculus rewrite: 
Lemma 1. Continuous matrix-valued semimartingales equipped with the left and right half-shuffles and the covariation bracket obey the axioms (5)-(11) together with

$$
[X,[Y, Z]]=[[X, Y], Z]=0
$$

Notice that the associativity of the product is formally a consequence of these axioms. This observation is of little interest when dealing with stochastic integrals for which the associativity of the product is somehow obvious. However, it is relevant with respect to the axiomatic point of view.

Definition 6. A regular quasi-shuffle algebra is a quasi-shuffle algebra such that the bracket satisfies the extra axiom (23).

The continuity hypothesis has more interesting consequences when dealing with Fisk-Stratonovich integrals. We follow closely the exposition in [25]. The Stratonovich formula is indeed then the usual integration by parts formula

$$
X_{t} Y_{t}=(X \geqslant Y)_{t}+(X \preccurlyeq Y)_{t},
$$

The classical statement that the Stratonovich integral for continuous semimartingales obeys the usual laws of calculus translates formally into the

Theorem 3. For continuous semimartingales $X, Y, Z$, the left and right Fisk-Stratonovich integrals satisfy the half-shuffle identities

$$
\begin{aligned}
& (X \preccurlyeq Y) \leqslant Z=X \preccurlyeq(Y Z) \\
& (X \geqslant Y) \leqslant Z=X \geqslant(Y \leqslant Z) \\
& X \geqslant(Y \geqslant Z)=(X Y) \geqslant Z .
\end{aligned}
$$

In particular, the algebra of continuous matrix-valued semimartingales is a noncommutative shuffle algebra.

Proof.

$$
\begin{aligned}
& (X \preccurlyeq Y) \leqslant Z=\left(X<Y+\frac{1}{2}[X, Y]\right) \leqslant Z \\
& =(X \prec Y)<Z+\frac{1}{2}[X, Y]<Z+\frac{1}{2}[X<Y, Z]+\frac{1}{4}[[X, Y], Z] \\
& =X \prec(Y Z)+\frac{1}{2}[X, Y<Z]+\frac{1}{2}[X, Y>Z] \\
& =X \preccurlyeq(Y Z) .
\end{aligned}
$$

Identity (27) is proved similarly.

$$
\begin{aligned}
& (X \geqslant Y) \leqslant Z=\left(X>Y+\frac{1}{2}[X, Y] \leqslant Z\right. \\
& =(X>Y)<Z+\frac{1}{2}[X, Y]<Z+\frac{1}{2}[X>Y, Z]+\frac{1}{4}[[X, Y], Z]
\end{aligned}
$$




$$
\begin{aligned}
& =X>(Y \prec Z)+\frac{1}{2}[X, Y \prec Z]+\frac{1}{2} X>[Y, Z] \\
& =X \geqslant(Y \preccurlyeq Z) .
\end{aligned}
$$

In general, the same argument show

Theorem 4. The map $(A, \prec,>,[],) \longmapsto(A, \preccurlyeq, \geqslant)$ is a functor from the category of regular quasi-shuffle algebras to the category of shuffle algebras.

\section{Chronological calculus for stochastic integration}

In this section, the second part of this work, we start by briefly reviewing the classical chronological calculus following Agrachev, Gamkrelidze and collaborators $[1,2,3,4]$. The aim is to show how chronological calculus can be applied in the context of stochastic calculus. The key idea is to use the notion of pre-Lie (or chronological) algebra instead of that of usual Lie algebra, to analyse groupand Lie-theoretical phenomena associated to evolution equations. We refer to [34] where this point of view is developed in more detail.

\subsection{Chronological calculus and pre-Lie algebra}

Time- or path-ordered products are ubiquitous, especially in theoretical physics and control theory, and form the basis for Agrachev and Gamkrelidze's chronological calculus [1]. These authors understood that the combination of Lie algebra and integration by parts permits to define the useful notion of chronological algebra [2], better known as pre-Lie or Vinberg algebra in algebra and geometry [13, 17, 61]. Concepts from chronological calculus apply in the context of stochastic integration as far as iterated Stratonovich integrals for continuous semimartingales are concerned, because they obey the usual rules of calculus.

However, for Itô and Stratonovich integrals in the non-continuous case, the usual ideas of chronological calculus do not apply immediately, due to the terms arising from the jump component of the covariation bracket. It turns out that in this case, one must appeal to results originating in the study of non-commutative Rota-Baxter algebras. We refer to [34] for more details as well as to joint works [25, 33] for results in that direction related to stochastic exponentials in the context of Itô calculus.

In a nutshell, chronological calculus is based on the idea of time-ordering of operators. Consider for example two time-dependent operators, $M(t)$ and $N(t)$ (with $M(0)=N(0)=0$ ), in a non-unital algebra $A$ of operators - having suitable regularity properties allowing to compute derivatives, integrals, and so on. The classical integration by parts rule is satisfied 


$$
\begin{aligned}
M(t) N(t) & =\int_{0}^{t} d s \int_{0}^{s} d u \dot{M}(s) \dot{N}(u)+\int_{0}^{t} d s \int_{0}^{s} d u \dot{M}(u) \dot{N}(s) \\
& =:(M>N)(t)+(M \prec N)(t) .
\end{aligned}
$$

We recognise in $<$ and $>$ the usual operations of left/right integration - restricted to the context of deterministic processes. In particular, they satisfy the shuffle algebra axioms (16). Agrachev and Gamkrelidze observed that the binary operation

$$
(M \triangleright N)(t):=(M>N)(t)-(N \prec n)(t)=\int_{0}^{t} d s \int_{0}^{s} d u[\dot{M}(s), \dot{N}(u)]
$$

has particular properties defining a chronological algebra structure on $A$. The latter is known as pre-Lie or Vinberg algebra in the mathematical literature.

Consider a vector space $A$ with a binary product $\triangleright: A \otimes A \rightarrow A$ and the associated bracket product $[a, b]_{\triangleright}:=a \triangleright b-b \triangleright a$. Write $L_{x}$ for the linear endomorphism of $A$ defined by left multiplication, $L_{x}(y):=x \triangleright y$, and define the usual commutator bracket of linear endomorphisms of $A,\left[L_{x}, L_{y}\right]_{\circ}:=L_{x} \circ L_{y}-L_{y} \circ L_{x}$.

Definition 7 ([2]). The pair $(A, \triangleright)$ is a pre-Lie algebra if and only if for any $x, y \in A$, the identity $\left[L_{x}, L_{y}\right]_{\odot}=L_{[x, y]_{\triangleright}}$ holds, which is equivalent to the (left) pre-Lie relation

$$
x \triangleright(y \triangleright z)-(x \triangleright y) \triangleright z=y \triangleright(x \triangleright z)-(y \triangleright x) \triangleright z .
$$

The notion of pre-Lie algebra is finer than that of Lie algebra (it contains more information). Indeed, pre-Lie algebras are Lie admissible, that is, if $A$ is a pre-Lie algebra, then $\left(A,[-,-]_{\triangleright}\right)$ is a Lie algebra.

The link with classical chronological calculus is as follows. Consider an algebra $\mathscr{A}$ of matrix-valued continuous semimartingales equipped with the left/right FiskStratonovich integrals, $\geqslant$ and $\leqslant$, defined in (20) respectively (21). We write $\llbracket-,-\rrbracket$ for its commutator bracket:

$$
\llbracket X, Y \rrbracket_{t}:=X_{t} Y_{t}-Y_{t} X_{t} .
$$

According to our previous developments, computing in this algebra amounts to computing with time-dependent operators. The - Fisk-Stratonovich - integration by parts formula implies that

$$
\llbracket X, Y \rrbracket_{t}=\int_{0}^{t} X_{s} \circ d Y_{s}+\int_{0}^{t} \circ d X_{t} Y_{t}-\int_{0}^{t} Y_{s} \circ d X_{t}-\int_{0}^{t} \circ d Y_{s} X_{s},
$$

which can be written as the difference of:

$$
(X \triangleright Y)_{t}:=(X \geqslant Y-Y \leqslant X)_{t}=\int_{0}^{t} X_{s} \circ d Y_{s}-\int_{0}^{t} \circ d Y_{s} X_{S}
$$

and $(Y \triangleright X)_{t}$ so that $\llbracket X, Y \rrbracket=[X, Y]_{\triangleright}$. That the algebra $\mathscr{A}$ is indeed a pre-Lie algebra, that is, 


$$
\left([X, Y]_{\triangleright} \triangleright Z\right)_{t}=(X \triangleright(Y \triangleright Z))_{t}-(Y \triangleright(X \triangleright Z))_{t} .
$$

follows from the Jacobi identity of the commutator bracket on the non-commutative algebra $\mathscr{A}$.

Remark 8. The same argument shows that, more generally, there is a forgetful functor from shuffle to pre-Lie algebras, that is, any shuffle algebra $(A, \prec,>)$ has the structure of a pre-Lie algebra with pre-Lie product: $x \triangleright y:=x>y-y<x$.

Let us apply these ideas in the context of stochastic exponentials. Recall a fundamental object in the classical analysis of differential equations, known as the Magnus formula [60] and its pre-Lie interpretation [2, 28]. It follows from studying the formal properties of the flow associated to a matrix differential equation using a Lie theoretic approach, for theoretical and numerical reasons. Consider for instance the evolution operator solution of the linear differential equation $\dot{X}(t)=X(t) H(t)$ with initial value $X(0)=\mathbf{1}$, the identity matrix. Its logarithm is computed by a Lie series. Truncating the expansion of this $\log$ arithm, $\Omega(t):=\log (X(t))$, and taking its exponential is a classical and efficient way to approximate $X(t)$ numerically, while preserving group-theoretic properties [10, 50].

The logarithm can be computed using the Baker-Campbell-Hausdorff formula (see, e.g., [64]) or Magnus' non-linear differential equation [60]

$$
\dot{\Omega}(t)=\frac{a d_{\Omega}}{e^{a d_{\Omega}-1}} H(t)=H(t)+\sum_{n>0} \frac{B_{n}}{n !} a d_{\Omega(t)}^{n}(H(t)),
$$

where $a d$ stands for the usual Lie adjoint representation, $\operatorname{ad}_{N}(M):=N M-M N$, $a d_{N}^{0}(M)=M$, and the $B_{n}$ are the Bernoulli numbers.

Let us explain how the formula adapts to Stratonovich integrals using recently developed algebraic tools that are most likely not familiar in the context of stochastic integration. The following developments are based on [34]. We omit here the group-theoretical perspective that relies on two underlying Hopf algebra structures, existing on the enveloping algebra of any pre-Lie algebra [20].

Let $\mathscr{A}$ be our usual algebra of continuous matrix-valued semimartingales, now equipped with the pre-Lie product $\triangleright$. The algebra of polynomials over $\mathscr{A}$ is denoted $\mathbb{R}[\mathscr{A}]$ and we identify $m$-multilinear maps symmetric in the $m$ entries with maps from the degree $m$ component of this polynomial algebra. To avoid confusion between the product of two matrix-valued semimartingales in $\mathscr{A}$ and their (commutative) product in $\mathbb{R}[\mathscr{A}]$, we denote the latter $X \odot Y$. The brace map on $\mathscr{A}$ is the family of symmetric multilinear maps into $\mathscr{A}$

$$
\mathbb{R}[\mathscr{A}] \otimes \mathscr{A} \longrightarrow \mathscr{A}, \quad P \otimes X \longmapsto\{P\} X,
$$

defined inductively by

$$
\{Y\} X:=Y \triangleright X,
$$

for $Y, X \in \mathscr{A}$, and for $Y_{1}, Y_{2}, \ldots, Y_{n}, X \in \mathscr{A}$ we have 


$$
\left\{Y_{1}, \ldots, Y_{n}\right\} X:=\left\{Y_{n}\right\}\left(\left\{Y_{1}, \ldots, Y_{n-1}\right\} X\right)-\sum_{i=1}^{n-1}\left\{Y_{1}, \ldots,\left\{Y_{n}\right\} Y_{i}, \ldots, Y_{n-1}\right\} X .
$$

Observe that for $n=2$ the last equality encodes the pre-Lie identity (29) as $\left\{Y_{1}, Y_{2}\right\} X=\left\{Y_{2}, Y_{1}\right\} X$. Following Guin and Oudom [69], we introduce a product * on $\mathbb{R}[\mathscr{A}]$ in terms of the brace map. For elements $X_{1}, \ldots, X_{n}$ and $Y_{1}, \ldots, Y_{m}$ in $\mathscr{A}$,

$$
\left(Y_{1} \odot \cdots \odot Y_{m}\right) *\left(X_{1} \odot \cdots \odot X_{n}\right):=\sum_{f} W_{0} \odot\left\{W_{1}\right\} X_{1} \odot \cdots \odot\left\{W_{n}\right\} X_{n}
$$

where the sum is over all maps $f$ from $\{1, \ldots, m\}$ to $\{0, \ldots, n\}$ and the $W_{i}:=$ $\prod_{j \in f^{-1}(i)} Y_{j}$. For example, $Y * X=Y X+\{Y\} X$, for $X, Y \in \mathscr{A}$.

Recall now that the enveloping algebra, $U(L)$, of a Lie algebra $L$ is an associative algebra (uniquely defined up to isomorphism) such that [72]:

- the Lie algebra $L$ embeds in $U(L)$ (as a Lie algebra, where the Lie algebra structure on $U(L)$ is induced by the associative product, that is, in terms of the ususal commutator bracket,

- for any associative algebra $A$ (which is a Lie algebra, $L_{A}$, when equipped with the commutator bracket), there is a natural bijection between Lie algebra maps from $L$ to $A$ and associative algebra maps from $U(L)$ to $A$.

The central result of the work of Oudom and Guin [69] is the next theorem.

Theorem 5 ([69]). $\mathbb{R}[\mathscr{A}]$ with the product $*$ defined in (30) is a non-commutative, associative and unital algebra. The product makes $\mathbb{R}[\mathscr{A}]$ the enveloping algebra of the Lie algebra $L_{\mathscr{A}}$ associated to $\mathscr{A}$.

Applying Theorem 5 to $\mathscr{A}$ we see that the commutator bracket in $\mathscr{A}$ identifies with the pre-Lie bracket: $\llbracket X, Y \rrbracket=[,]_{\triangleright}$. On the other hand, by the universal properties of enveloping algebras, there is a unique associative algebra map $\imath$ from $(\mathbb{R}[A], *)$ to $\mathscr{A}$ which is the identity on $\mathscr{A}$. In degree two we have:

$$
\begin{aligned}
\imath(Y \odot X)=\imath(Y * X)-\imath(\{Y\} X) & =Y X-Y \triangleright X \\
& =Y \leqslant X+Y \geqslant X-(Y \geqslant X-X \leqslant Y) \\
& =Y \leqslant X+X \leqslant Y=: \mathscr{T}\langle Y, X\rangle,
\end{aligned}
$$

where, using now the language of theoretical physics, we call time-ordered product of two elements in $\mathscr{A}$ the product $\mathscr{T}\langle Y, X\rangle:=X \leqslant Y+Y \leqslant X$. In general, for $X_{1}, \ldots, X_{n} \in \mathscr{A}$,

$$
\mathscr{T}\left\langle X_{1}, X_{2}, \ldots, X_{n}\right\rangle:=\sum_{\sigma \in S_{n}} X_{\sigma(1)} \preccurlyeq\left(X_{\sigma(2)} \preccurlyeq\left(\cdots \preccurlyeq\left(X_{\sigma(n-1)} \preccurlyeq X_{\sigma(n)}\right) \cdots\right)\right),
$$

where $S_{n}$ denotes the symmetric group of order $n$. The degree two calculation is a particular instance of a general phenomenon. The following Theorem relating preLie products and time-ordered exponentials was obtained in [31, p. 1291]: 
Theorem 6. The image in $\mathscr{A}$ of a monomial $X_{1} \odot \cdots \odot X_{n} \in \mathbb{R}[\mathscr{A}]$ by the canonical map 1 is the time-ordered product of the $X_{i}$ s in $\mathscr{A}$ :

$$
\imath\left(X_{1} \odot \cdots \odot X_{n}\right)=\mathscr{T}\left\langle X_{1}, \ldots, X_{n}\right\rangle .
$$

Notice that, in particular,

$$
\frac{1}{n !} l\left(X^{\odot n}\right)=\frac{1}{n !} \mathscr{T}\langle X, \ldots, X\rangle=X \preccurlyeq(X \preccurlyeq(\cdots \preccurlyeq(X \preccurlyeq X) \cdots)) .
$$

Let us apply these ideas to the study of the stochastic exponential and its logarithm in the Stratonovich framework. We address these problems at a purely formal level. Regarding the existence of the stochastic exponential and the convergence issues of the related series we refer to [70] for the Itô case and to Ben Arous [9] and Castell [18] for the Stratonovich one.

Definition 8. For a continuous matrix-valued semimartingale, $X \in \mathscr{A}$, the (Stratonovich) right stochastic exponential is defined through

$$
\mathscr{E}_{\leqslant}(X)=\mathbf{1}+\left(X \leqslant \mathscr{E}_{\leqslant}(X)\right),
$$

or, by a Picard iteration, as a series

$$
\mathscr{E}_{\leqslant}(X)=\mathbf{1}+X+X \leqslant X+\cdots+X \leqslant(X \leqslant(\cdots \leqslant(X \leqslant X) \cdots))+\cdots
$$

We are interested in the stochastic analogue of the classical Baker-CampbellHausdorff problem of computing the logarithm $\Omega(X)$ of the solution

$$
\Omega(X)=\log \left(\mathscr{E}_{\leqslant}(X)\right) .
$$

Since Stratonovich calculus obeys the usual integration by parts rule, the wellknown Strichartz formula holds $[9,64,77]$. We are interested here in the stochastic analog of the so-called Magnus solution.

By Theorem 6, the equation

$$
\mathscr{E}_{\leqslant}(X)=\exp (\Omega(X))
$$

lifts in $\mathbb{R}[\mathscr{A}]$ to an equality of exponentials:

$$
\exp ^{\odot}(X)=\exp ^{*}(\tilde{\Omega}(X)),
$$

where $\exp ^{\odot}(X)\left(\right.$ resp. $\left.\exp ^{*}(\tilde{\Omega}(X))\right)$ denotes the exponential of $X \in \mathscr{A}($ resp. $\tilde{\Omega}(X))$ for the $\odot($ resp. $*)$ product. Theorem 6 together with the general properties of enveloping algebras insure that this identity maps to (32) by $\imath$ and that $\imath(\tilde{\Omega}(X))=$ $\Omega(X)$. The next proposition was shown in [20], using the fact that the maps exp ${ }^{\odot}$ and $\exp ^{*}$ have a Lie theoretic interpretation.

Proposition 1. The element $\tilde{\Omega}(X)=\log ^{*} \circ \exp ^{\odot}(X)$ belongs to $\mathscr{A}$ and satifies the fixed point equation: 


$$
\tilde{\Omega}(X)=\left\{\frac{\tilde{\Omega}(X)}{\exp ^{*}(\tilde{\Omega}(X))-\mathbf{1}}\right\} X,
$$

where $\tilde{\Omega}(X) /\left(\exp ^{*}(\tilde{\Omega}(X))-\mathbf{1}\right)$ is computed in $\mathbb{R}[\mathscr{A}]$ using the * product.

Note that the brace map is in place on the righthand side in (34). We set

$$
\ell_{X \triangleright}^{(n)}(Y):=X \triangleright\left(\ell_{X \triangleright}^{(n-1)}(Y)\right), \ell_{X \triangleright}^{(0)}(Y):=Y .
$$

The $B_{n} / n$ ! are the coefficients of the formal power series expansion of $x /(\exp (x)-1)$ and, by formal properties of the enveloping algebra, we have $\imath\left(\left\{X^{* n}\right\} Y\right)=\ell_{X \triangleright}^{(n)}(Y)$. We refer, e.g., to [34] for an explanation of this general phenomenon in the context of enveloping algebras of pre-Lie algebras. We recover finally the pre-Lie Magnus expansion of the logarithm of the right Stratonovich exponential obtained in [25].

Theorem 7. The continuous matrix-valued semimartingale $\Omega(X)$ satisfies the fixed point equation

$$
\Omega(X)=\sum_{n \geqslant 0} \frac{B_{n}}{n !} \ell_{\Omega(X) \triangleright}^{(n)}(X) .
$$

Remark 9. The left stochastic exponential is defined similarly through $\mathscr{E} \geqslant(X)_{t}=$ $\mathbf{1}+(\mathscr{E} \geqslant(X) \geqslant X)_{t}$. It satisfies

$$
\mathscr{E} \geqslant(X)=\exp (-\Omega(-X))
$$

\subsection{Chronological Itô calculus}

In the present subsection we will apply the machinery developed in the previous subsection in the context of Itô calculus. As remarked earlier, our arguments are purely algebraic. We do neither address the question of existence of stochastic exponentials nor do we discuss convergence issues of the associated series. On these questions, the reader is referred to the standard reference [70].

The existence of a continuous Baker-Campbell-Hausdorff, or Strichartz formula, the presence of pre-Lie structures as well as a Magnus formula could be expected in Stratonovich calculus due to the fact that the latter satisfies the usual rules of calculus. In Itô calculus, however, things are not so simple due to the presence of the covariation bracket and the fact that the usual shuffle algebra structure must be replaced by Karandikar's axioms, i.e., a quasi-shuffle algebra. For iterated Itô integrals of matrix-valued semimartingales, a Strichartz-type formula was obtained in $[32,33]$. The difference with the classical formula reflect the fact that one must take into account the covariation bracket. This is achieved by replacing bijections, that is, permutations and their descent statistics as they appear in the classical formula, by surjections and a suitable notion of descents in this new context.

Here, we focus again on pre-Lie structures and the Magnus formula in the context of Itô calculus. Our results are obtained by adapting ideas from the theory of Rota- 
Baxter algebras to quasi-shuffle algebras. Our presentation is almost self-contained. On Rota-Baxter algebras and integral calculus, we refer to [34] for a general survey combined with references.

In this subsection, $\mathscr{A}$ denotes an algebra of matrix-valued semimartingales (notice that we do not require continuity anymore).

Proposition 2. For $X, Y \in \mathscr{A}$, set $X>Y:=X>Y+[X, Y]$ and $X \gg Y:=X \gg Y-Y<$ $X$, then the pair $(\mathscr{A}, \bullet)$ is a pre-Lie algebra. Moreover, $\llbracket X, Y \rrbracket=[X, Y]$.

Proof. Indeed, the quasi-shuffle axioms imply that

$$
X Y=X \prec Y+X>Y+[X, Y]=X \prec Y+X>Y .
$$

This yields

$$
\begin{aligned}
\llbracket X, Y \rrbracket=X Y-Y X & =X<Y+X>Y-Y<X-Y>X \\
& =(X>Y-Y<X)-(Y>X-X<Y) \\
& =X \bullet Y-Y \bullet X=[X, Y] \triangleright .
\end{aligned}
$$

Using the Jacobi identity (to avoid any notational ambiguity, recall that $[$,$] denotes$ in this article the covariation bracket, not to be confused with the Lie bracket $\llbracket, \rrbracket$ ):

$$
\begin{aligned}
& ([X, Y] \bullet \cdot Z)_{t}=(\llbracket X, Y \rrbracket \bullet Z)_{t}=\int_{0}^{t} \llbracket \llbracket X_{S}, Y_{s} \rrbracket, \circ d Z_{S} \rrbracket+[\llbracket X, Y \rrbracket, Z]_{t} \\
& =\left(\int_{0}^{t} \llbracket X_{s}, \llbracket Y_{S}, \circ d Z_{s} \rrbracket \rrbracket+[X Y, Z]_{t}\right)-\left(\int_{0}^{t} \llbracket Y_{S}, \llbracket X_{s}, \circ d Z_{s} \rrbracket \rrbracket+[X Y, Z]_{t}\right) \\
& =\int_{0}^{t} \llbracket X_{s}, \llbracket Y_{S}, \circ d Z_{s} \rrbracket \rrbracket+[X>Y+X<Y+[X, Y], Z]_{t} \\
& -\left(\int_{0}^{t} \llbracket Y_{S}, \llbracket X_{S}, \circ d Z_{s} \rrbracket \rrbracket+[Y>X+Y<X+[Y, X], Z]_{t}\right) \\
& =\int_{0}^{t} \llbracket X_{s}, \llbracket Y_{s}, \circ d Z_{s} \rrbracket \rrbracket+[X, Y>Z]_{t}+(X>[Y, Z])_{t}+[[X, Y], Z]_{t} \\
& -\left(\int_{0}^{t} \llbracket Y_{S}, \llbracket X_{S}, \circ d Z_{s} \rrbracket \rrbracket+[Y, X>Z]_{t}+(Y>[X, Z])_{t}+[[Y, X], Z]_{t}\right) \\
& =(X \bullet(Y \bullet Z))_{t}-(Y \bullet(X \bullet Z))_{t} .
\end{aligned}
$$

Remark 10. The triple $(\mathscr{A},<, \gg)$ is a shuffle algebra. More generally, in [27] it was shown that any quasi-shuffle algebra gives rise to a shuffle algebra. Indeed, $(X \prec$ $Y)<Z=X<(Y Z)$ and

$$
\begin{aligned}
(X>Y)<Z & =(X>Y)<Z+[X, Y]<Z \\
& =X>(Y<Z)+[X, Y<Z]=X>(Y \prec Z) \\
(X Y)>Z & =(X Y)>Z+[X Y, Z]
\end{aligned}
$$




$$
\begin{aligned}
& =(X Y)>Z+[X>Y, Z]+[X<Y, Z]+[[X, Y], Z] \\
& =X>(Y>Z)+X>[Y, Z]+[X, Y>Z]+[X,[Y, Z]] \\
& =X>(Y>Z)+[X, Y>Z]=X>(Y>Z) .
\end{aligned}
$$

We note that this property is also common in Rota-Baxter algebras, where a shuffle algebra structure is defined similarly starting from the operations $R(X) Y, X R(Y)$ and $X Y$ instead of $<,>,[-,-]$.

Definition 9. For a matrix-valued semimartingale $X \in \mathscr{A}$ the (Itô) right stochastic exponential is defined through

$$
\mathscr{E}_{<}(X)=\mathbf{1}+\left(X<\mathscr{E}_{<}(X)\right),
$$

or, by a Picard iteration, as a series

$$
\mathscr{E}_{<}(X)=\mathbf{1}+X+X<X+\cdots+X<(X \prec(\cdots \prec(X<X) \cdots))+\ldots
$$

We are interested again in the stochastic analogue of the Baker-CampbellHausdorff problem of computing the logarithm $\Gamma(X)$ of the solution:

$$
\mathscr{E}_{<}(X)=\exp (\Gamma(X)) .
$$

Let us denote now by $\mathbb{R},[\mathscr{A}]$ the enveloping algebra of $\mathscr{A}$ constructed exactly as in the previous section but using the new pre-Lie product $\bullet$ instead of $\triangleright$. In particular, as a vector space, $\mathbb{R}_{\bullet}[\mathscr{A}]=\mathbb{R}[\mathscr{A}]$, the algebra of polynomials over $\mathscr{A}$.

To avoid notational ambiguities, we write $*$, for the associative product making $\mathbb{R}_{\triangleright}[\mathscr{A}]$ the enveloping algebra of $L_{\mathscr{A}}$ and $\{P\}_{\diamond} X$ the brace map for $P \in \mathbb{R}_{\bullet}[\mathscr{A}]$. We also write $\mathscr{T}$ for the corresponding time-ordered product, associated to the left-half shuffle $<$, e.g., $\mathscr{T}[X, Y]:=X<Y$, and so on. Lastly, we denote $\imath_{\text {, the canoni- }}$ cal algebra map from $\left(\mathbb{R}_{\bullet}[\mathscr{A}], *_{\bullet}\right)$ to $\mathscr{A}$ obtained from the universal properties of the enveloping algebra. Theorem 6 holds mutatis mutandis in the new context and equation (37) lifts in $\mathbb{R}_{\bullet}[\mathscr{A}]$ to:

$$
\exp ^{\odot}(X)=\exp ^{* \bullet}(\tilde{\Gamma}(X))
$$

Theorem 6 and the general properties of enveloping algebras insure that this identity maps to (37) by $\iota_{\boldsymbol{}}$ and that $\iota_{\boldsymbol{}}(\tilde{\Gamma}(X))=\Gamma(X)$.

We obtain finally the analogous of Proposition 1 :

Proposition 3. The element $\tilde{\Gamma}(X)=\log ^{*} \circ \exp ^{\odot}(X)$ belongs to $\mathscr{A}$ and satifies the fixed point equation:

$$
\tilde{\Gamma}(X)=\left\{\frac{\tilde{\Gamma}(X)}{\exp *(\tilde{\Gamma}(X))-\mathbf{1}}\right\}, X,
$$

where $\tilde{\Gamma}(X) /\left(\exp _{\bullet}^{*}(\tilde{\Gamma}(X))-\mathbf{1}\right)$ is computed in $\mathbb{R}_{\bullet}[\mathscr{A}]$ using the $*_{\bullet}$ product.

Setting 


$$
\ell_{X \triangleright}^{(n)}(Y):=X \bullet\left(\ell_{X \bullet}^{(n-1)}(Y)\right)
$$

$\ell_{X}^{(0)}(Y):=Y$, we get finally a pre-Lie Magnus expansion of the logarithm of the right Itô stochastic exponential:

Theorem 8. The matrix-valued semi-martingale $\Gamma(X)$, which is the logarithm of the (Itô) stochastic exponential, satisfies the fixed point equation

$$
\Gamma(X)=\sum_{n \geqslant 0} \frac{B_{n}}{n !} \ell_{\Gamma(X)}^{(n)}(X)
$$

\section{References}

1. A. Agrachev, R. Gamkrelidze, The exponential representation of flows and chronological calculus, Math. sbornik 107 (149), (1978) 467-532; English transl. in Math. USSR Sbornik 35, (1979) 727-785.

2. A. Agrachev, R. Gamkrelidze, Chronological algebras and nonstationary vector fields, J. Sov. Math. 17, (1981) 1650-1675.

3. A. Agrachev, R. Gamkrelidze, V. Sarychev, Local Invariants of Smooth Control Systems, Acta Applicandae Mathematicae 14, (1989) 191-237.

4. A. Agrachev, Y. Sachkov, Control Theory from the Geometric Viewpoint, Encyclopaedia of Mathematical Sciences 84, Springer-Verlag Berlin Heidelberg 2004.

5. A. Agrachev, D. Barilari, U. Boscain, A Comprehensive Introduction to Sub-Riemannian Geometry, Cambridge Studies in Advanced Mathematics 181, Cambridge University Press 2019.

6. J. Baldeaux, E. Platen, Functionals of Multidimensional Diffusions with Applications to Finance, in Bocconi \& Springer Series 5 Springer 2013.

7. G. Baxter, An analytic problem whose solution follows from a simple algebraic identity, Pacific J. Math. 10, (1960) 731-742.

8. P. Beasley Cohen, T. W. M. Eyre, R. L. Hudson, Higher order Itô product formula and generators of evolutions and flows, Int. J. Theor. Phys. 34, (1995) 1-6.

9. G. Ben Arous, Flots et séries de Taylor stochastiques, Probab. Th. Rel. Fields 81, (1989) 29-77.

10. S. Blanes, F. Casas, J.A. Oteo, J. Ros, Magnus expansion: mathematical study and physical applications, Phys. Rep. 470, (2009) 151-238.

11. Ch. Brouder, Runge-Kutta methods and renormalization, Europ. Phys. J. C12, (2000) 512-534.

12. Y. Bruned, M. Hairer, L. Zambotti, Algebraic renormalisation of regularity structures, Invent. Math. 215(3), (2019) 1039-1156.

13. D. Burde, Left-symmetric algebras, or pre-Lie algebras in geometry and physics, Central European Journal of Mathematics 4 Nr. 3, (2006) 323-357.

14. E. Burgunder, M. Ronco, Tridendriform structure on combinatorial Hopf algebras, J. Algebra 324 (10), (2010) 2860-2883.

15. D. Calaque, K. Ebrahimi-Fard, D. Manchon, Two interacting Hopf algebras of trees: A Hopf-algebraic approach to composition and substitution of B-series, Advances in Applied Mathematics 47, (2011) 282-308.

16. P. Cartier, On the structure of free Baxter algebras, Advances in Mathematics 9 Issue 2, (1972) 253-265.

17. P. Cartier, Vinberg algebras, Lie groups and combinatorics, Clay Mathematical Proceedings 11, (2011) 107-126. 
18. F. Castell, Asymptotic expansion of stochastic flows, Probab. Th. Rel. Fields 96, (1993) 225-239.

19. F. Chapoton, M. Livernet, Pre-Lie algebras and the rooted trees operad, International Mathematics Research Notices 2001, (2001) 395-408.

20. F. Chapoton, F. Patras, Enveloping algebras of preLie algebras, Solomon idempotents and the Magnus formula, Int. J. Algebra and Computation 23 No. 4, (2013) 853-861.

21. K.T. Chen, Integration of paths, geometric invariants and a generalized Baker-Hausdorff formula, Ann. of Math. 65, (1957) 163-178.

22. K.T. Chen, Algebras of iterated path integrals and fundamental groups, Trans. Am. Math. Soc. 156, (1971) 359-379.

23. A. Connes, D. Kreimer, Hopf algebras, Renormalization and Noncommutative Geometry, Comm. Math. Phys. 199, (1998) 203-242.

24. C. Curry, K. Ebrahimi-Fard, S.J.A. Malham, A. Wiese, Lévy processes and quasi-shuffle algebras, Stochastics 86(4), (2014) 632-642.

25. C. Curry, K. Ebrahimi-Fard, F. Patras, On non-commutative stochastic exponentials, in proceedings volume ENUMATH2017 conference, Springer's Lecture Notes in Computational Science and Engineering, 126, 2018.

26. C. Curry, K. Ebrahimi-Fard, S.J.A. Malham, A. Wiese, Algebraic Structures and Stochastic Differential Equations driven by Lévy processes, Proc. R. Soc. A 475, (2019) 20180567.

27. K. Ebrahimi-Fard, Loday-type algebras and the Rota-Baxter relation, Lett. Math. Phys. 61(2), (2002) 139-147.

28. K. Ebrahimi-Fard, D. Manchon, A Magnus- and Fer-type formula in dendriform algebras, Found. Comput. Math. 9, (2009) 295-316.

29. K. Ebrahimi-Fard, A. Lundervold, S.J.A. Malham, H. Munthe-Kaas, A. Wiese, Algebraic structure of stochastic expansions and efficient simulation, Proc. R. Soc. A (2012).

30. K. Ebrahimi-Fard, D. Manchon, The tridendriform structure of a discrete Magnus expansion, Discrete and Continuous Dynamical Systems-A 34(3), (2014) 1021-1040.

31. K. Ebrahimi-Fard, F. Patras, The Pre-Lie Structure of the Time-Ordered Exponential, Lett. Math. Phys. 104, (2014) 1281-1302.

32. K. Ebrahimi-Fard, S.J.A. Malham, F. Patras, A. Wiese, The exponential Lie series for continuous semimartingales, Proc. R. Soc. A 471, (2015) 20150429.

33. K. Ebrahimi-Fard, S.J.A. Malham, F. Patras, A. Wiese, Flows and stochastic Taylor series in Ito calculus, J. Phys. A: Math. Theor. 48, (2015) 495202.

34. K. Ebrahimi-Fard, F. Patras, From iterated integrals and chronological calculus to Hopf and Rota-Baxter algebras, Encyclopedia in Algebra and Applications (to appear) arXiv:1911.08766.

35. F. Fauvet, F. Menous, Ecalle's arborification-coarborification transforms and ConnesKreimer Hopf algebra, Ann. Sci. Éc. Norm. Supér. 50, (2017) 39-83.

36. L. Foissy, F. Patras, Lie theory for quasi-shuffle bialgebras, in Periods in Quantum Field Theory and Arithmetic, (Burgos Gil. et al., eds) Springer Proceedings in Mathematics and Statistics 314, 2020.

37. L. Foissy, F. Patras, J.-Y. Thibon, Deformations of shuffles and quasi-shuffles, Annales Inst. Fourier 66 no 1, (2016) 209-237.

38. R. Friedrich, Operads in Itô calculus, arXiv:1604.08547.

39. J. Gaines, The algebra of iterated stochastic integrals, Stochast. and Stochast. Rep. 49, (1994) 169-179.

40. M. Gubinelli, Ramification of rough paths, J. Differential Equations 248, (2010) 693721.

41. M. Gubinelli, Abstract integration, Combinatorics of Trees and Differential Equations, in Proceedings of the Workshop Combinatorics and Physics, 2007. MPI Bonn. Combinatorics and physics, 135-151, Contemp. Math., 539, Amer. Math. Soc., Providence, RI, 2011. 
42. E. Hairer, C. Lubich, G. Wanner, Geometric numerical integration Structure-preserving algorithms for ordinary differential equations, vol. 31, Springer Series in Computational Mathematics. Springer-Verlag, Berlin, 2002.

43. M. Hairer, Solving the KPZ equation, Annals of Mathematics 178, (2013) 559-664.

44. M. Hairer, A theory of regularity structures, Invent. Math. 198(2), (2014) 269-504.

45. M. Hairer, D. Kelly, Geometric versus non-geometric rough paths, Annales de l'I.H.P. Probabilités et Statistiques 51(1), (2015) 207-251.

46. M. E. Hoffman, Quasi-Shuffle Products, J. Algebr. Combinator. 11, no. 1, (2000) 49-68.

47. M. E. Hoffman, K. Ihara, Quasi-shuffle products revisited, J. Algebra 481 1, (2017) 293 326.

48. R. L. Hudson, Hopf-algebraic aspects of iterated stochastic integrals, Infinite Dimensional Analysis, Quantum Probability and Related Topics 12, (2009) 479-496.

49. R. L. Hudson, Sticky shuffle product Hopf algebras and their stochastic representations, in "New trends in stochastic analysis and related topics", Interdiscip. Math. Sci. 12, (2012) 165-181, World Sci. Publ., Hackensack, NJ.

50. A. Iserles, H. Z. Munthe-Kaas, S. P. Nørsett and A. Zanna, Lie-group methods, Acta Numer. 9 (2000), 215-365.

51. R. L. Karandikar, A.s. approximation results for multiplicative stochastic integration, Séminaire de Probabilitiés XVI. Lecture Notes in Math. 920, 384-391, Springer, Berlin (1981).

52. R. L. Karandikar, Multiplicative decomposition of non-singular matrix valued continuous semimartingales, Ann. Probab. 10, (1982) 1088-1091.

53. R. L. Karandikar, Girsanov type formula for a lie group valued brownian motion, Séminaire de Probabilitiés XVII. Lecture Notes in Math. 986 198-204, Springer, Berlin (1982).

54. R. L. Karandikar, Multiplicative decomposition of nonsingular matrix valued semimartingales, in Séminaire de Probabilités XXV, Lecture Notes in Math. 1485, (J. Azéma, M. Yor, P. Meyer, eds.), Springer, Berlin, 1991, 262-269.

55. J.-L. Loday, M. Ronco, Une dualité entre simplexes standards et polytopes de Stasheff, C. R. Acad. Sci. Paris Série I 333, (2001) 81-86.

56. A. Lundervold, H. Z. Munthe-Kaas, Hopf algebras of formal diffeomorphisms and numerical integration on manifolds, Contemporary Mathematics 539, (2011) 295-324

57. T. Lyons, Differential equations driven by rough signals, Rev. Mat. Iberoamericana 14(2), (1998) 215-310.

58. T. Lyons, M. J. Caruana, T. Lévy, Differential Equations Driven by Rough Paths, Ecole d'Eté de Probabilités de Saint-Flour XXXIV-2004 1908, Springer-Verlag Berlin Heidelberg 2007.

59. R. I. McLachlan, K. Modin, H. Munthe-Kaas, O. Verdier, Butcher Series - A Story of Rooted Trees and Numerical Methods for Evolution Equations, Asia Pacific Mathematics Newsletter 7 No 1, (2017) 1-11.

60. W. Magnus, On the exponential solution of differential equations for a linear operator, Commun. Pure Appl. Math. 7, (1954) 649-673.

61. D. Manchon, A short survey on pre-Lie algebras, in E. Schrödinger Institut Lectures in Math. Phys., Non-commutative Geometry and Physics: Renormalisation, Motives, Index Theory (A. Carey, ed.), EMS 2011.

62. F. Menous, F. Patras, Renormalization: a quasi-shuffle approach, in Computation and Combinatorics in Dynamics, Stochastics and Control: The Abel Symposium 2016, (Celledoni et al., eds) Springer Abel Symposia 132018.

63. F. Menous, F. Patras, Right-handed bialgebras and the Prelie forest formula, Annales I.H.P. Série D, 5, Issue 1, (2018) 103-125.

64. B. Mielnik, J. Plebański, Combinatorial approach to Baker-Campbell-Hausdorff exponents, Ann. Inst. Henri Poincaré A XII, (1970) 215-254.

65. H. Z. Munthe-Kaas, W. M. Wright, On the Hopf algebraic structure of Lie group integrators, Foundations of Computational Mathematics 8(2), (2007) 227-257 
66. A. Murua, The Hopf algebra of rooted trees, free Lie algebras, and Lie series, Found. Comput. Math. 6, (2006) 387-426.

67. J.-C. Novelli, F. Patras, J.-Y. Thibon, Natural endomorphisms of quasi-shuffle Hopf algebras, Bull. Soc. Math. France 141, (2013) 107-130.

68. J.-C. Novelli, J.-Y. Thibon, Polynomial realizations of some trialgebras, Proceedings of Formal Power Series and Algebraic Combinatorics, San Diego, California, 2006.

69. J.-M. Oudom, D. Guin, On the Lie enveloping algebra of a pre-Lie algebra, Journal of K-theory 2, issue 1, (2008) 147-167.

70. P. E. Protter, Stochastic Integration and Differential Equations, Version 2.1, 2nd Edn., Springer, Berlin (2005).

71. R. Ree, Lie Elements and an Algebra Associated With Shuffles, Ann. of Math., Second Series 68, no. 2, (1958) 210-220.

72. C. Reutenauer, Free Lie algebras, Oxford University Press (1993).

73. G.-C. Rota, Baxter algebras and combinatorial identities. I, II, Bull. Amer. Math. Soc. 75, (1969) 325-329; ibid. 75, (1969) 330-334.

74. G.-C. Rota, D. Smith, Fluctuation theory and Baxter algebras, Istituto Nazionale di Alta Matematica IX, (1972) 179.

75. G.-C. Rota, Baxter operators, an introduction, in: "Gian-Carlo Rota on Combinatorics, Introductory papers and commentaries", J. P. S. Kung Ed., Contemp. Mathematicians, Birkhäuser Boston, Boston, MA 1995.

76. G.-C. Rota, Ten mathematics problems I will never solve, Invited address at the joint meeting of the American Mathematical Society and the Mexican Mathematical Society, Oaxaca, Mexico, Dec. 6, 1997. DMV Mittellungen Heft 2, (1998) 45.

77. R. S. Strichartz, The Campbell-Baker-Hausdorff-Dynkin formula and solutions of differential equations, J. Func. Anal. 72, (1987) 320-345. 